\title{
Chemical Constituents and Antimicrobial Activity of Branches and Leaves of Cordia insignis (Boraginaceae)
}

\author{
Correia, F. C. S.; Targanski, S. K.; Bomfim. T. R. D.; da Silva, Y. S. A. D.; Violante, I. \\ M. P.; de Carvalho, M. G.; Sousa Jr, P. T.; Silva, V. C. P.; Ribeiro, T. A. N.*
}

Rev. Virtual Quim., 2020, 12 (3), 809-816. Data de publicação na Web: 29 de Maio de 2020

http://rvq.sbq.org.br

\section{Constituintes Químicos e Atividade Antimicrobiana de Ramos e Folhas de Cordia insignis (Boraginaceae)}

Resumo: A investigação fitoquímica dos galhos e folhas da espécie Cordia insignis levou ao isolamento e identificação de $\mathbf{6}$-sitosterol (1), estigmasterol (2), campesterol (3), 3-O-6-D-glicopiranosil sitosterol (4), 3-O-6-D- glicopiranosil estigmasterol (5), escoparona (6), $\alpha$-amirina (7), $\beta$-amirina (8), cafeoato de metila (9), alantoína (10), escopoletina (11), ácido cafeico (12) e rosmarinato de metila (13). A determinação estrutural foi feita através de dados de RMN (1D e 2D) e CG-EM e comparando com os dados da literatura. Os extratos hidroetanólicos dos galhos e folhas e suas respectivas frações foram submetidos à avaliação antibacteriana e antifúngica. Os extratos e frações apresentaram boa atividade antifúngica, porém não apresentaram atividade antibacteriana. Este estudo é o primeiro trabalho com a espécie $C$. insignis.

Palavras-chave: Cordia insignis; metabólitos secundários; atividade antifúngica.

\begin{abstract}
Phytochemical investigation of the branches and leaves of the species Cordia insignis led to the isolation and identification of $\boldsymbol{B}$-sitosterol (1), stigmasterol (2), campesterol (3), $\beta$-sitosterol 3-O- $\beta$-D-glucopyranoside (4), stigmasterol 3-O- $\beta$-D-glucopyranoside (5), scoparone (6), $\alpha$-amyrin (7), $\beta$-amyrin (8), methyl caffeate (9), allantoin (10), scopoletin (11), caffeic acid (12), methyl rosmarinate (13). These substances had their structures determined by analysis of their 1D and 2D NMR and GC-MS spectra and by comparison with data reported in the literature. The hydroethanolic extracts of the branches and leaves and their respective fractions were submitted to antibacterial and antifungal evaluation. The extracts and fractions showed good antifungal activity but did not present antibacterial activity. This study is the first work with $C$. insignis.
\end{abstract}

Keywords: Cordia insignis; secundary metabolites; antifungal activity.

* Universidade Federal de Mato Grosso, Departamento de Química, Av. Fernando Corrêa da Costa 2367, CEP 78060-900, Cuiabá-MT, Brasil.

1terezaribeiro20@gmail.com DOI: $10.21577 / 1984-6835.20200063$ 


\section{Chemical Constituents and Antimicrobial Activity of Branches and Leaves of Cordia insignis (Boraginaceae)}

\section{Francyne C. S. Correia, ${ }^{a}$ Sabrina K. Targanski, ${ }^{a}$ Tamires R. D. Bomfim, ${ }^{a}$ Yasmin S.} A. D. da Silva, ${ }^{b}$ Ivana M. P. Violante, ${ }^{b}$ Mario G. de Carvalho, ${ }^{c}$ Paulo T. de Sousa Jr, ${ }^{a}$ Virgínia C. P. Silva, ${ }^{a}$ Tereza A. N. Ribeiro ${ }^{\mathrm{a}, *}$

a Universidade Federal de Mato Grosso, Departamento de Química, Avenida Fernando Corrêa da Costa 2367, CEP 78060-900, Cuiabá-MT, Brasil.

b Universidade de Cuiabá, Centro de Pesquisa da Faculdade de Farmácia-Bioquímica, Rua José de Arruda 3100, CEP 78065-900, Cuiabá-MT, Brasil.

c Universidade Federal Rural do Rio de Janeiro, Instituto de Química, BR 465, km 07, CEP 23890-000, Seropédica-RJ, Brasil.

*terezaribeiro20@gmail.com

Recebido em 13 de Maio de 2019. Aceito para publicação em 19 de Maio de 2020.

\section{Introduction}

\section{Experimental}

2.1. General procedures

2.2. Plant material

2.3. Extraction and isolation

2.4. Antimicrobial assay

\section{Results and Discussion}

3.1. Isolated compounds

3.2. Antimicrobial bioassay

\section{Conclusion}

\section{Introduction}

The genus Cordia (Boraginaceae) which comprise of 320 species found in temperate and subtropical regions, but mainly in South America. They are distributed throughout Brazilian territory, with emphasis on the Atlantic and Cerrado forest biomes. ${ }^{1}$ In several Cordia species the following classes of metabolites were identified: triterpenes, flavonoids, saponins, quinones, terpenes, alkaloids, coumarins, phenyl propanoids, etc. ${ }^{1,2}$, ${ }^{3}$ The major pharmacological activities reported for extracts and isolated compounds include anti-inflammatory, antioxidant, larvicidal, hepatoprotective, analgesic, antimicrobial and antidiabetic. ${ }^{4}$ The methanolic extract of branches and leaves of $C$. salicifolia presented cytotoxic activity against cancer cells. ${ }^{5}$ Aqueous and hydroalcoholic extract of $C$. americana were found to be the most effective against herpes simplex virus type $1 .{ }^{6}$ The methanol extract of the bark $C$. platythyrsa showed significant antiinflammatory effect in carrageenan-induced rat paw edema. ${ }^{7}$ Ethanol bark extract of $C$. rothii demonstrated a significant hypoglycemic effect 
when estimating fasting and fed blood glucose levels using Dextrostix kit. ${ }^{8}$ The Cordia insignis species, popularly known as "calção-de-velho", is restricted to Brazil and is distributed in the Northeast, Midwest and Southeast regions in Cerrado environments. ${ }^{9}$ In folk medicine its leaves are used in the form of tea or bottled in the treatment of general pains and rheumatism. ${ }^{10}$ The present work is the first report about the chemical constituents and antibacterial and antifungal activities in vitro of $C$. insignis.

\section{Experimental}

\subsection{General Procedures}

All compounds were identified by one and twodimensional Nuclear Magnetic Resonance - 1D and 2D NMR and/or Gas Chromatography Mass Spectrometry experiments and in comparison, with the literature. NMR experiments were acquired in $\mathrm{CDCl}_{3}$, methanol- $d_{4}$ or DMSO- $d_{6}$ at $293 \mathrm{~K}$ on Bruker AM-500 500 NMR spectrometer operating at 14.1 Tesla, observing ${ }^{1} \mathrm{H}$ and ${ }^{13} \mathrm{C}$ at $500.13 \mathrm{MHz}$ and $150.76 \mathrm{MHz}$, respectively, equipped with a $5-\mathrm{mm}$ quadrinuclear inverse detection probe with z-gradient. Chemical shifts $(\delta)$ were expressed in ppm and coupling constants $(J)$ in Hertz. NMR spectra were referenced by TMS. GC-MS were performed on a Shimadzu CGMS 5050 chromatograph, employing DB5 $(30 \mathrm{~m} / 0,25$ $\mathrm{mm}$ ) column (JW). Silica gel 60 (70-230 mesh) was used for column chromatography (CC) with 0.063-0.200 mm particles (Merck), while silica gel 60 F254 was used for thin-layer chromatography (TLC).

\subsection{Plant Material}

Cordia insignis (CGEN code AF8CO57) was collected in Chapada dos Guimarães, Mato Gosso State, Brazil in March 2014 (S 15'21.964' W $\left.055^{\circ} 57.372^{\prime}\right)$. A voucher specimen (41454) is available at the UFMT Central Herbarium (Cuiabá, Mato Grosso State, Brazil).

\subsection{Extraction and isolation}

Dried and ground branches $(2.12 \mathrm{~kg})$ and leaves $(1.26 \mathrm{~kg})$ of $C$. insignis were extracted separately with $\mathrm{EtOH} / \mathrm{H}_{2} \mathrm{O}(80: 20)$ at room temperature. After concentration under reduced pressure were obtained hydroethanolic extract of the branches HEB (185.21 g) and leaves HEL (166.72 g). HEB $(140.0 \mathrm{~g})$ was suspended in $250 \mathrm{~mL}$ of $\mathrm{MeOH}$ : $\mathrm{H}_{2} \mathrm{O}(80: 20)$ and extracted with $n$-hexane (FHB, $7.73 \mathrm{~g}), \mathrm{CHCl}_{3}(\mathrm{FCB}, 8.38 \mathrm{~g})$, EtOAc (FAB, $38.60 \mathrm{~g}$ ) and the hydromethanolic residue (FHMB, 84.25). The HEL $(156.0 \mathrm{~g})$ was solubilized in $500 \mathrm{~mL}$ of $\mathrm{MeOH}$ and $500 \mathrm{~mL}$ of distilled water and allowed to stand in the refrigerator for $48 \mathrm{~h}$. After the solution was filtered to remove the chlorophyll. The hydromethanolic solution was extracted with n-hexane (FHL, $0.25 \mathrm{~g}$ ), $\mathrm{CHCl}_{3}$ ( $\mathrm{FCL}, 3.95 \mathrm{~g}$ ), EtOAc (FAL, $14.23 \mathrm{~g}$ ) and the hydromethanolic residue (FHML, $98.3 \mathrm{~g}$ ). All fractions, except FHL (low mass), were submitted to chromatography on silica gel (70-230 mesh) using $n-\mathrm{C}_{6} \mathrm{H}_{14}, \mathrm{CHCl}_{3}$ or $\mathrm{CH}_{2} \mathrm{Cl}_{2}, \mathrm{EtOAC}$ and $\mathrm{MeOH}$ (pure or in increasing gradient of solvent polarity) and Sephadex LH20 using $\mathrm{MeOH}$ or $\mathrm{CHCl}_{3}$ (pure or in mixture). FHB (5.5 g) to afford mixture 1, 2 and $\mathbf{3}(7.2 \mathrm{mg})$ through elution with $n-\mathrm{C}_{6} \mathrm{H}_{14}$-EtOAc (9:1) and mixture 4 and 5 (10.2 mg) on elution in $n-\mathrm{C}_{6} \mathrm{H}_{14}{ }^{-}$ EtOAc (2:8). FCB $(6.45 \mathrm{~g})$ to give $6(3 \mathrm{mg})$ from the elution $\mathrm{CHCl}_{3}$-EtOAC (7:3). FAB (30.2 g) was subjected to $\mathrm{CC}$ on silica gel eluted $\mathrm{CHCl}_{3}$-EtOAC and EtOAc-MeOH to obtain 8 fractions. Fraction 1 (421.3 mg) from the elution $\mathrm{CHCl}_{3}$-EtOAc (7:3) was purified on Sephadex LH-20 column eluted with $\mathrm{CHCl}_{3}$ to provide mixture of 7 and $8(5.3 \mathrm{mg})$. Fraction 6 (1.98 g) was separated by $\mathrm{CC}\left(\mathrm{SiO}_{2}\right.$; $\mathrm{CHCl}_{3}$ - EtOAc and EtOAc-MeOH) to give 9 (1.4 mg) through elution with $\mathrm{CHCl}_{3}-\mathrm{EtOAC}$ (3:7). Fraction 7 $(18.14 \mathrm{~g})$ was submitted to $\mathrm{CC}\left(\mathrm{SiO}_{2}\right.$; EtOAc-MeOH) to give $10(15.8 \mathrm{mg})$ on elution in EtOAc:MeOH (8:2). FCL (3.0 g) was subjected to CC (gradient $\mathrm{CH}_{2} \mathrm{Cl}_{2}$-EtOAc and EtOAc-MeOH) obtained 7 fractions. Fraction $3(16.5 \mathrm{mg})$ was separated by preparative $\mathrm{TLC}\left(\mathrm{CH}_{2} \mathrm{Cl}_{2}\right.$-EtOAc, 9:1) to yield 6 (4.5 $\mathrm{mg}$ ) and $\mathbf{1 1}(5 \mathrm{mg})$. FAL (12 g) was subjected to CC $\left(\mathrm{SiO}_{2}\right.$, gradient EtOAc-MeOH) to afford 9 fractions. Fraction $3(123 \mathrm{mg}$ ) eluted on EtOAc was purified by Sephadex $\mathrm{LH} 20 \mathrm{CC}$ with $\mathrm{MeOH}$ yielding 12 (7.1 $\mathrm{mg}$ ) and mixture of $\mathbf{1 2}$ and $\mathbf{1 3}(10.2 \mathrm{mg})$. Fraction 6 to give $\mathbf{1 0}$ (15 mg) in EtOAc: $\mathrm{MeOH}(8: 2)$.

\subsection{Antimicrobial assay}

For the bioassays of the antimicrobial activity standardized strains were used originating from the American Type Culture Collection (ATCC) 
against six standard fungal strains: Candida albicans (ATCC 90028), C. glabrata (ATCC 9030), C. krusei (ATCC 6258), C. parapsilosis (ATCC 22019), C. tropicalis (ATCC 760) and Cryptococcus neoformans (ATCC 32045); five Gram-positive bacterial strains: Enterococcus faecalis (ATCC 29212), Staphylococcus aureus (ATCC 25923), Staphylococcus epidermidis (ATCC 12228), Streptococcus pyogenes (ATCC) 19615, Bacillus subitilis (6051), and five Gramnegative bacterial strains: Escherichia coli (ATCC 25922), Pseudomonas aeruginosa (ATCC 27853), Klebsiela pneumoniae (ATCC 700603), Salmonella typhimurium (ATCC 14028) and Shigella flexneri (ATCC 12022). Amphotericin $B$ and Chloramphenicol were used as the reference antimycotic and antibacterial controls, respectively. The antifungal and antibacterial activities of extracts and fractions of $C$. insignis were determined using microbroth dilution assays in 96 -well microplates, in duplicate. ${ }^{11,12}$ The antimicrobial activity was detected using a colorimetric method by adding $25 \mu \mathrm{L}$ of the resazurin staining $(0.01 \%)$ aqueous solution in each well at the end of the incubation period. ${ }^{13,14}$ The minimal inhibitory concentration (MIC) was defined as the lowest extract concentration able to inhibit the microbial growth, as indicated by resazurin staining.

\section{Results and Discussion}

\subsection{Isolated compounds}

Chemical investigation of leaves and branches of $C$. insignis resulted in the isolation and identification of 13 compounds (Figure 1), including: three steroids, $\beta$-sitosterol (1), stigmasterol (2), campesterol (3), ${ }^{15}$ two saponins $\beta$-sitosterol 3-O- $\beta$-D-glucopyranoside (4), stigmasterol 3-O- $\beta$-D-glucopyranoside (5), 16,17 two coumarins, scoparone (6), ${ }^{18}$ scopoletin (11), ${ }^{19}$ two triterpenes $\alpha$-amyrin (7), $\beta$-amyrin (8), ${ }^{20}$ three phenylpropanoic derivates methyl caffeate (9), ${ }^{21}$ caffeic acid (12), ${ }^{22}$ methyl rosmarinate (13), ${ }^{23}$ and a ureide allantoin $(\mathbf{1 0})^{24}$ were identified by $1 \mathrm{D}$ and 2D NMR and GC-MS spectra and by comparison with the reported data in the literature.

6-sitosterol (1): ${ }^{1} \mathrm{H}-\mathrm{NMR}\left(400 \mathrm{MHz}, \mathrm{CDCl}_{3}\right) \delta$ : 3.51-3.57 (1H, $m, \mathrm{H}-3), 5.36(1 \mathrm{H}, s l, \mathrm{H}-6) ;{ }^{13} \mathrm{C}-\mathrm{NMR}$ $\left(125 \mathrm{MHz}, \mathrm{CDCl}_{3}\right)$ ): 37.2 (C-1), 31.6 (C-2), 71.8 (C-3), 42.3 (C-4), 140.7 (C-5), 121.6 (C-6), 31.6 (C-7,8), 50.2 (C-9), 36.1 (C-10), 21.1 (C-11), 39.7 (C-12), 42.3 (C-13), 56.8 (C-14), 24.2 (C-15), 28.2 (C-16), 56.0 (C-17), 12.1 (C-18), 19.3 (C-19), 40.4 (C-20), 18.7 (C-21), 33.9 (C-22), 26.1 (C-23), 45.9 (C-24), 29.6 (C-25), 19.7 (C-26), 19.0 (C-27), 23.1 (C-28), 11.9 (C-29); GC-MS: $m / z$ L14 $\left[\mathrm{C}_{29} \mathrm{H}_{50} \mathrm{O}\right]$.

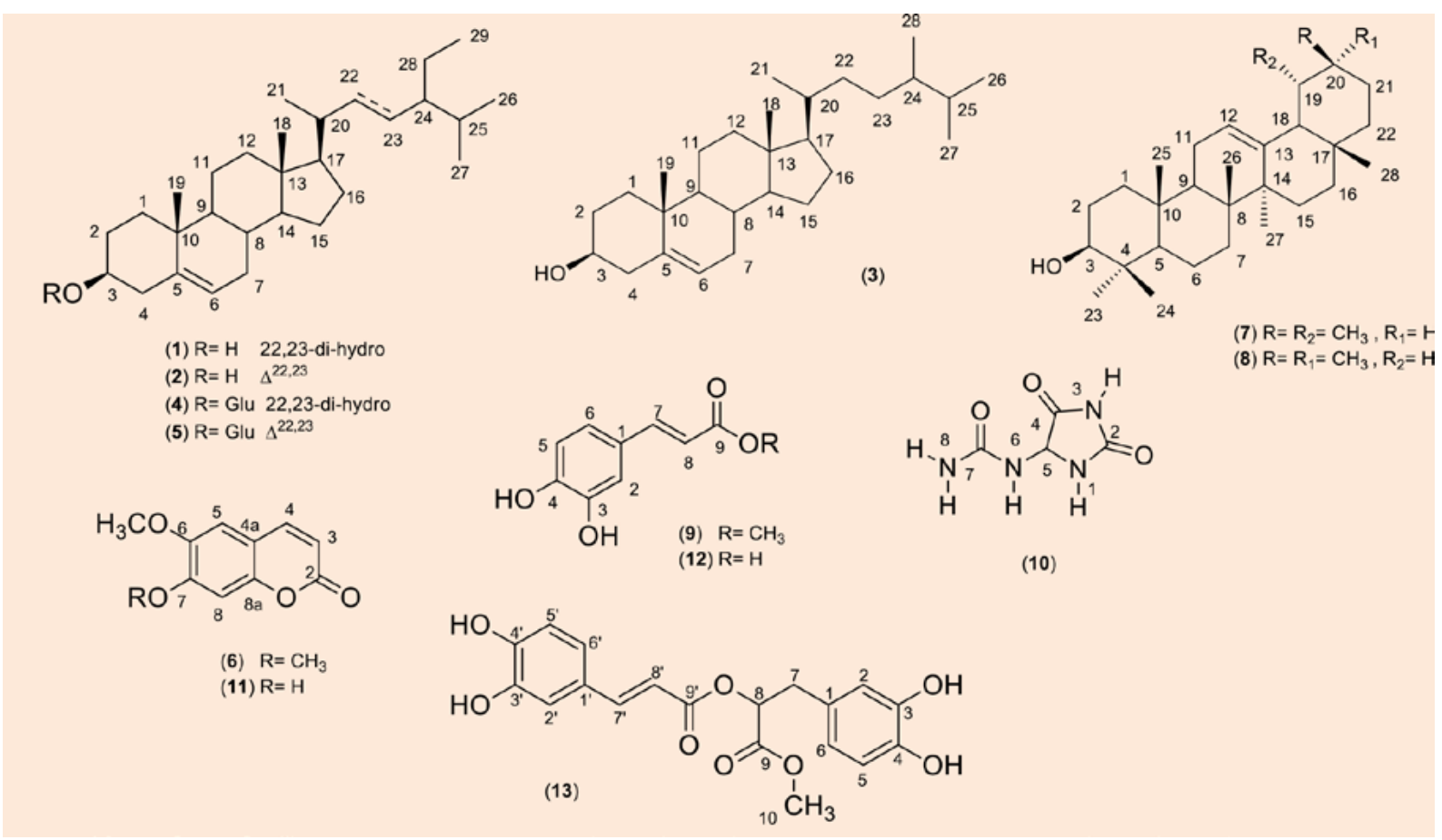

Figure 1. Structures of isolated compounds of Cordia insignis 
Stigmasterol (2): ${ }^{1} \mathrm{H}-\mathrm{NMR}\left(400 \mathrm{MHz}, \mathrm{CDCl}_{3}\right) \delta$ : 3.51-3.57 (1H, $m, \mathrm{H}-3), 5.36(1 \mathrm{H}, s l, \mathrm{H}-6), 5.18(1 \mathrm{H}$, $d d, \mathrm{~J}=15.2,8.6 \mathrm{~Hz}, \mathrm{H}-22), 5.05(1 \mathrm{H}, d d, \mathrm{~J}=15.2$, $8.6 \mathrm{~Hz}, \mathrm{H}-23) ;{ }^{13} \mathrm{C}-\mathrm{NMR}\left(125 \mathrm{MHz}, \mathrm{CDCl}_{3}\right) \delta: 37.2$ (C-1), 31.6 (C-2), 71.8 (C-3), 42.3 (C-4), 140.7 (C-5), 121.6 (C-6), 31.6 (C-7,8), 50.2 (C-9), 36.1 (C-10), 21.1 (C-11), 39.7 (C-12), 42.3 (C-13), 56.8 (C-14), 24.2 (C-15), 28.2 (C-16), 56.0 (C-17), 12.1 (C-18), 19.3 (C-19), 40.4 (C-20), 20.1 (C-21), 138.2 (C-22), 129.3 (C-23), 50.1 (C-24), 31.9 (C-25), 22.6 (C-26), 19.7 (C-27), 25.3 (C-28), 11.8 (C-29). GC-MS: $\mathrm{m} / \mathrm{z}$ $412\left[\mathrm{C}_{29} \mathrm{H}_{48} \mathrm{O}\right]$

Campesterol (3): ${ }^{1} \mathrm{H}-\mathrm{NMR}\left(400 \mathrm{MHz}, \mathrm{CDCl}_{3}\right) \delta$ : 3.51-3.57 (1H, $m, \mathrm{H}-3), 5.36(1 \mathrm{H}, s l, \mathrm{H}-6) ;{ }^{13} \mathrm{C}-\mathrm{NMR}$ $\left(125 \mathrm{MHz}, \mathrm{CDCl}_{3}\right.$ ) 8: 37.2 (C-1), 31.6 (C-2), 71.8 (C-3), 42.3 (C-4), 140.7 (C-5), 121.6 (C-6), 31.6 (C-7,8), 50.2 (C-9), 36.1 (C-10), 21.1 (C-11), 39.7 (C-12), 42.3 (C-13), 56.8 (C-14), 24.2 (C-15), 28.2 (C-16), 56.0 (C-17), 12.1 (C-18), 19.3 (C-19), 40.4 (C-20), 18.7 (C-21), 33.9 (C-22), 26.1 (C-23), 45.9 (C-24), 29.6 (C-25), 19.7 (C-26), 19.0 (C-27), 23.1 (C-28). GC-MS: $m / z 400\left[\mathrm{C}_{28} \mathrm{H}_{48} \mathrm{O}\right]$

$\beta$-sitosterol 3-O- $\beta$-D-glucopyranoside (4): ${ }^{1} \mathrm{H}-\mathrm{NMR}\left(400 \mathrm{MHz}, \mathrm{C}_{5} \mathrm{D}_{5} \mathrm{~N}\right)$ 8: 3.82-3.91 $(1 \mathrm{H}, \mathrm{m}$, $\mathrm{H}-3), 5.35(1 \mathrm{H}, s l, \mathrm{H}-6), 4.87(1 \mathrm{H}, d l, \mathrm{~J}=7.7 \mathrm{~Hz}$, $\left.\mathrm{H}-1^{\prime}\right), 3.98-4.05\left(2 \mathrm{H}, \mathrm{m}, \mathrm{H}-2^{\prime}, 3^{\prime}\right), 4.32-4.38(2 \mathrm{H}$, $\left.m, \mathrm{H}-4^{\prime}, 5^{\prime}\right), 4.17(2 \mathrm{H}, d d, \mathrm{~J}=11.7,5.4 \mathrm{~Hz}), \mathrm{H}-6^{\prime}$; ${ }^{13} \mathrm{C}-\mathrm{NMR}\left(125 \mathrm{MHz}, \mathrm{C}_{5} \mathrm{D}_{5} \mathrm{~N}\right)$ ) $8: 37.2$ (C-1), 30.0 (C2), 78.2 (C-3), 42.2 (C-4), 140.7 (C-5), 121.7 (C6), 31.9 (C-7), 31.8 (C-8), 50.1 (C-9), 36.7 (C-10), 21.0 (C-11), 39.1 (C-12), 42.1 (C-13), 56.6 (C-14), 24.2 (C-15), 28.3 (C-16), 56.0 (C-17), 11.7 (C-18), 19.0 (C-19), 36.1 (C-20), 18.8 (C-21), 33.9 (C-22), 26.1 (C-23), 45.8 (C-24), 29.2 (C-25), 19.7 (C-26), 19.2 (C-27), 23.1 (C-28), 11.9 (C-29), 102.3 (C-1'), 75.0 (C-2'), 77.9 (C-3'), 71.4 (C-4'), 78.3 (C-5'), $62.6\left(C-6^{\prime}\right)$.

Stigmasterol 3-0-6-D-glucopyranoside (5): ${ }^{1} \mathrm{H}-\mathrm{NMR}\left(400 \mathrm{MHz}, \mathrm{C}_{5} \mathrm{D}_{5} \mathrm{~N}\right) \delta: 3.82-3.91(1 \mathrm{H}, \mathrm{m}$, $\mathrm{H}-3), 5.35(1 \mathrm{H}, s \mathrm{l}, \mathrm{H}-6), 5.20(1 \mathrm{H}, d d, \mathrm{~J}=15.1,8.7$ $\mathrm{Hz}, \mathrm{H}-22), 5.05(1 \mathrm{H}, d d, \mathrm{~J}=15.1,8.7 \mathrm{~Hz}, \mathrm{H}-23)$, 4.58-3.60 (6H, $\left.m, \mathrm{H}^{\prime} \mathbf{1}^{\prime}, 2^{\prime}, 3^{\prime}, 4^{\prime}, 5^{\prime}, 6^{\prime}\right):{ }^{13} \mathrm{C}-\mathrm{NMR}(125$ $\left.\mathrm{MHz}, \mathrm{C}_{5} \mathrm{D}_{5} \mathrm{~N}\right)$ 8: 37.2 (C-1), $30.0(\mathrm{C}-2), 78.2(\mathrm{C}-3)$, 42.2 (C-4), 140.7 (C-5), 121.7 (C-6), 31.9 (C-7), 31.8 (C-8), 50.1 (C-9), 36.7 (C-10), 21.0 (C-11), 39.1 (C12), 42.1 (C-13), 56.6 (C-14), 24.2 (C-15), 28.3 (C16), 56.0 (C-17), 11.7 (C-18), 19.0 (C-19), 36.1 (C20), 18.8 (C-21), 138.6 (C-22), 129.2 (C-23), 45.8 (C-24), 29.2 (C-25), 19.7 (C-26), 19.2 (C-27), 23.1 (C-28), 11.9 (C-29), 102.3 (C-1'), 75.0 (C-2'), 77.9 (C-3'), 71.4 (C-4'), $78.3\left(\mathrm{C}-5^{\prime}\right), 62.6$ (C-6').
Scoparone (6): ${ }^{1} \mathrm{H}-\mathrm{NMR}\left(500 \mathrm{MHz}, \mathrm{CDCl}_{3}\right.$ ) $\delta$ : $6.32(1 \mathrm{H}, d, \mathrm{~J}=9.45 \mathrm{~Hz}, \mathrm{H}-3), 7.65(1 \mathrm{H}, d, \mathrm{~J}=9.45$ $\mathrm{Hz}, \mathrm{H}-4), 6.83(1 \mathrm{H}, \mathrm{s}, \mathrm{H}-5), 7.82(1 \mathrm{H}, \mathrm{s}, \mathrm{H}-8), 3.95$ $\left(3 \mathrm{H}, s,-\mathrm{OCH}_{3}\right), 3.98\left(3 \mathrm{H}, s,-\mathrm{OCH}_{3}\right)$; DEPT $135(125$ $\mathrm{MHz}, \mathrm{CDCl}_{3}$ ) $\delta: 161.4$ (C-2), 113.5 (C-3), 143.3 (C4), 111.4 (C-4a), 107.9 (C-5), 146.3 (C-6), 152.8 (C-7), 100.0 (C-8), 150.0 (C-8a), $56.4\left(-\mathrm{OCH}_{3}\right), 56.3$ $\left(-\mathrm{OCH}_{3}\right)$. GC-MS: $m / z 206\left[\mathrm{C}_{11} \mathrm{H}_{10} \mathrm{O}_{4}\right]$.

$\boldsymbol{\alpha}$-amyrin (7): DEPT 135 (125 MHz, CD $\mathrm{OD}) \delta$ : 38.4 (C-1), 28.3 (C-2), 78.3 (C-3), 38.9 (C-4), 55.3 (C-5), 18.1 (C-6), 32.4 (C-7), 39.1 (C-8), 48.4 (C-9), 36.7 (C-10), 23.1 (C-11), 125.7 (C-12), 138.5 (C13), 41.4 (C-14), 26.5 (C-15), 26.4 (C-16), 33.5 (C17), 55.5 (C-18), 41.3 (C-19, 20), 30.9 (C-21), 41.8 (C-22), 27.3 (C-23), 15.2 (C-24), 15.0 (C-25), 16.3 (C-26), 22.5 (C-27), 27.3 (C-28), 17.1 (C-29), 20.8 (C-30).

$\boldsymbol{\beta}$-amyrin (8): DEPT 135 (125 MHz, CD $\mathrm{OD}) \delta$ : 38.4 (C-1), 27.4 (C-2), 78.2 (C-3), 38.9 (C-4), 55.3 (C-5), 18.0 (C-6), 32.4 (C-7), 39.1 (C-8), 48.4 (C-9), 36.6 (C-10), 23.1 (C-11), 122.2 (C-12), 143.8 (C13), 41.4 (C-14), 26.1 (C-15, 16), 32.6 (C-17), 46.2 (C-18), 45.8 (C-19), 31.6 (C-20), 34.5 (C-21), 38.7 (C-22), 27.3 (C-23), 14.9 (C-24), 14.4 (C-25), 16.3 (C-26), 24.9 (C-27), 27.3 (C-28), 34.2 (C-29), 22.5 (C-30).

Methyl caffeate (9): ${ }^{1} \mathrm{H}-\mathrm{NMR}(500 \mathrm{MHz}$, $\left.\mathrm{CD}_{3} \mathrm{OD}\right) \delta: 7.05(1 \mathrm{H}, d, \mathrm{~J}=2.0 \mathrm{~Hz}, \mathrm{H}-2), 6.79(1 \mathrm{H}, \mathrm{d}$, $\mathrm{J}=8.2 \mathrm{~Hz}, \mathrm{H}-5), 6.96(1 \mathrm{H}, d d, \mathrm{~J}=2.0,8.2 \mathrm{~Hz}, \mathrm{H}-6)$, $7.56(1 \mathrm{H}, d, \mathrm{~J}=15.9 \mathrm{~Hz}, \mathrm{H}-7), 6.28(1 \mathrm{H}, d, \mathrm{~J}=15.9$ $\mathrm{Hz}, \mathrm{H}-8), 3.78(3 \mathrm{H}, \mathrm{s}) ;{ }^{13} \mathrm{C}-\mathrm{NMR}\left(125 \mathrm{MHz}, \mathrm{CD}_{3} \mathrm{OD}\right)$

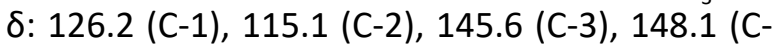
4), 113.4 (C-5), 121.6 (C-6), 145.3 (C-7), 113.7 (C8), 168.5 (C-9), $56.9\left(-\mathrm{OCH}_{3}\right)$.

Allantoin (10): ${ }^{1} \mathrm{H}-\mathrm{NMR}\left(500 \mathrm{MHz}\right.$, DMSO- $\left.d_{6}\right) \delta$ : $8.04(1 \mathrm{H}, s \mathrm{l}, \mathrm{H}-1), 10.53(1 \mathrm{H}, \mathrm{s}, \mathrm{H}-3), 5.25(1 \mathrm{H}, d, \mathrm{~J}=$ $8.1 \mathrm{~Hz}, \mathrm{H}-5), 6.89(1 \mathrm{H}, d, \mathrm{~J}=8.1 \mathrm{~Hz}, \mathrm{H}-6), 5.77(1 \mathrm{H}$, $s l, \mathrm{H}-8) ;{ }^{13} \mathrm{C}-\mathrm{NMR}\left(125 \mathrm{MHz}\right.$, DMSO-d $\left.d_{6}\right) \delta: 156.8$ (C2), 157.4 (C-4), 62.9 (C-5), 174.1 (C-7).

Scopoletin (11): ${ }^{1} \mathrm{H}-\mathrm{NMR}\left(500 \mathrm{MHz}, \mathrm{CD}_{3} \mathrm{OD}\right) \delta$ : $6.26(1 \mathrm{H}, d, J=9.4 \mathrm{~Hz}, \mathrm{H}-3), 7.84(1 \mathrm{H}, d, \mathrm{~J}=9.4 \mathrm{~Hz}$, $\mathrm{H}-4), 6.98(1 \mathrm{H}, \mathrm{s}, \mathrm{H}-5), 8.50(1 \mathrm{H}, \mathrm{sl},-\mathrm{OH}), 6.99(1 \mathrm{H}$, $s, \mathrm{H}-8), 3.97\left(3 \mathrm{H}, \mathrm{s},-\mathrm{OCH}_{3}\right) ;{ }^{13} \mathrm{C}-\mathrm{NMR}(125 \mathrm{MHz}$, $\left.\mathrm{CD}_{3} \mathrm{OD}\right)$ 8: 168.8 (C-2), 112.1 (C-3), 143.9 (C-4), 111.5 (C-4a), 104.2 (C-5), 144.4 (C-6), 152.7 (C-7), 99.2 (C-8), 148.9 (C-8a), $55.4\left(-\mathrm{OCH}_{3}\right)$.

Caffeic acid (12): ${ }^{1} \mathrm{H}-\mathrm{NMR}\left(500 \mathrm{MHz}, \mathrm{CD}_{3} \mathrm{OD}\right) \delta$ : $7.05(1 \mathrm{H}, d, J=2.0 \mathrm{~Hz}, \mathrm{H}-2), 6.79(1 \mathrm{H}, d, \mathrm{~J}=8.2 \mathrm{~Hz}$, $\mathrm{H}-5), 6.94(1 \mathrm{H}, d d, \mathrm{~J}=8.2,2.0 \mathrm{~Hz}, \mathrm{H}-6), 7.53(1 \mathrm{H}$, $d, \mathrm{~J}=15.9 \mathrm{~Hz}, \mathrm{H}-7), 6.24(1 \mathrm{H}, d, \mathrm{~J}=15.9 \mathrm{~Hz}, \mathrm{H}-8)$; 
${ }^{13} \mathrm{C}-\mathrm{NMR}\left(125 \mathrm{MHz}, \mathrm{CD}_{3} \mathrm{OD}\right)$ 8: 126.5 (C-1), 113.6 (C-2), 145.1 (C-3), 145.3 (C-4), 115.0 (C-5), 121.3 (C-6), 147.9 (C-7), 116.3 (C-8), 170.0 (C-9).

Methyl rosmarinate (13): ${ }^{1} \mathrm{H}-\mathrm{NMR}(500 \mathrm{MHz}$, $\left.\mathrm{CD}_{3} \mathrm{OD}\right)$ 8: $7.07\left(1 \mathrm{H}, d, \mathrm{~J}=2.1 \mathrm{~Hz}, \mathrm{H}-2^{\prime}\right), 6.79(1 \mathrm{H}, d$, $\left.\mathrm{J}=8.1 \mathrm{~Hz}, \mathrm{H}-5^{\prime}\right), 6.98\left(1 \mathrm{H}, d d, \mathrm{~J}=8.3,2.0 \mathrm{~Hz}, \mathrm{H}-6^{\prime}\right)$, $7.57\left(1 \mathrm{H}, d, \mathrm{~J}=15.9 \mathrm{~Hz}, \mathrm{H}-7^{\prime}\right), 6.28(1 \mathrm{H}, d, \mathrm{~J}=15.9$ $\left.\mathrm{Hz}, \mathrm{H}-8^{\prime}\right), 5.21(2 \mathrm{H}, d d, \mathrm{~J}=7.6,5.5 \mathrm{~Hz}, \mathrm{H}-8), 3.03$ $(1 \mathrm{H}, d d, \mathrm{~J}=14.4,7.7 \mathrm{~Hz}, \mathrm{H}-7 \mathrm{a}), 3.08(1 \mathrm{H}, d d, \mathrm{~J}=$ $14.8,5.8 \mathrm{~Hz}, \mathrm{H}-7 \mathrm{~b}), 6.59(1 \mathrm{H}, d d, \mathrm{~J}=8.1,2.1 \mathrm{~Hz}$, $\mathrm{H}-6), 6.72(1 \mathrm{H}, d, \mathrm{~J}=8.1 \mathrm{~Hz}, \mathrm{H}-5), 6.73(\mathrm{~d}, \mathrm{~J}=2.1$ $\mathrm{Hz}, \mathrm{H}-2) 3.72(3 \mathrm{H}, s) .{ }^{13} \mathrm{C}-\mathrm{NMR}\left(125 \mathrm{MHz}, \mathrm{CD}_{3} \mathrm{OD}\right)$

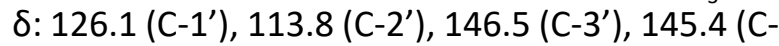
$\left.4^{\prime}\right), 115.1$ (C-5'), 121.8 (C-6'), 148.4 (C-7'), 112.7 (C-8'), 166.9 (C-9'), 170.7 (C-9), 73.2 (C-8), 36.5 (C-7), 120.4 (C-6), 115.0 (C-5), 144.8 (C-4), 143.9 (C-3), 116.1 (C-2), $127.3(\mathrm{C}-1), 51.2\left(-\mathrm{OCH}_{3}\right)$.

\subsection{Antimicrobial bioassay}

The crude hydroethanolic extracts were evaluated against the fungal and bacterial strains described in this work. It can be observed that no extract or fraction showed promising activity against the bacterial strains. For the fungal strains (Table 1), the extract of leaves (HEL) and fractions $n$-hexane (FHL), $\mathrm{CHCl}_{3}(\mathrm{FCL})$ and ethyl acetate (FAL) were active against $C$. albicans, $C$. glabrata and $C$. krusei strains, with MIC ranging from 15.62 to $250 \mu \mathrm{g} / \mathrm{mL}$. The FHL, FCL and FAL fractions also presented significant results against the $C$. neoformans strain, with MIC ranging from 125 to
$500 \mu \mathrm{g} / \mathrm{mL}$. The extract of branches (HEB) and $\mathrm{CHCl}_{3}$ (FCB) fraction presented promising results against the $C$. albicans strain, with MIC of 500 and $125 \mu \mathrm{g} / \mathrm{mL}$, respectively. The ethyl acetate (FCB) fraction was also active against $C$. glabrata strains with MIC $500 \mu \mathrm{g} / \mathrm{mL}$. These promising results may be related to the presence of substances that present antifungal activities.

\section{Conclusion}

The phytochemical investigation of Cordia insignis extracts led to the isolation and identification of thirteen compounds. The present work is the first report about the chemical constituents and antibacterial and antifungal activities in vitro of $C$. insignis. These substances are in accordance with the chemistry of the genus Cordia and these results suggest that $C$. insignis provides initial evidence for a new and alternative source of substances of medicinal interest with respect to antifungal activity.

\section{Acknowledgment}

The authors are grateful to INCT-Áreas Úmidas/CNPq/CPP (process no. 573990/2008-5), FAPEMAT (Universal 5/2015 e 0220269/2017), CAPES for scholarships and financial support.

Table 1. Antifungal activity of crude extract and fractions from C. insignis (MIC $\mu \mathrm{g} / \mathrm{mL}$ )

\begin{tabular}{lcccccc}
\hline Extracts/fractions & C. albicans & C. glabrata & C. krusei & C. parapsilosis & C. tropicalis & C. neoformans \\
\hline HEB & 500 & - & - & - & - & - \\
FHG & - & - & - & - & - & - \\
FCB & 125 & 500 & - & - & - & - \\
FAB & - & - & - & - & - & - \\
FHMB & - & 500 & 250 & - & - & - \\
HEL & 250 & 250 & 125 & - & - & - \\
FHL & 15.6 & 500 & 500 & - & - & 500 \\
FCL & 125 & 125 & 250 & - & - & 250 \\
FAL & 62.5 & - & - & - & - & - \\
FHML & - & 0.25 & 0.50 & 0.25 & 0.25 & 0.25 \\
AMP B & 0.50 & & & - & - \\
\hline
\end{tabular}

(-) No activity; AMP B: amphotericin B 


\section{References}

${ }^{1}$ Nogueira, T. B. S. S.; Nogueira, R. B. S. S.; Silva D. A.; Tavares, J. F.; Lima, E. O.; Pereira, F. O; Fernandes, M. M. M. S.; de Medeiros, F. A.; Sarquis, R. S. F. R.; Braz-Filho, R.; Maciel, J. K. S.; de Souza, M. F. $V$. First chemical constituents from Cordia exaltata Lam and antimicrobial activity of two neolignans. Molecules 2013, 18, 11086. [CrossRef] [PubMed] ${ }^{2}$ De Oliveira, F. M.; Silva Júnior, H. M.; Viana, F. A.; Diniz, J. C.; Rocha, S. A. S. Quantificação por CLAE de naftoquinonas do extrato das raízes de Cordia leucocephala Moric. Holos 2012, 1, 41. [CrossRef] ${ }^{3}$ Da Silva, S. A. S.; Agra, M. F.; Tavares, J. F.; da Cunha, E. V. L.; Barbosa-Filho, J. M.; da Silva, M. S. Flavanones from aerial parts of Cordia globosa (Jacq.) Kunth, Boraginaceae. Revista Brasileira de Farmacognosia 2010, 20, 682. [CrossRef]

${ }_{4}^{4}$ Oza, M. J.; Kulkarni, Y. A. Traditional uses, phytochemistryand pharmacology of themedicinal species of the genus Cordia (Boraginaceae). Journal of Pharmacy and Pharmacology 2017, 69, 755. [CrossRef] [PubMed]

${ }^{5}$ Siqueira, V. L. D.; Cortez, D. A. G.; de Oliveira, C. E.; Nakamura, C. V.; Bazotte, R. B. Pharmacological studies of Cordia salicifolia Cham in normal and diabetic rats. Brazilian Archives of Biology and Technology 2006, 49, 215. [CrossRef]

${ }^{6}$ Moura-Costa, G. F.; Nocchi, S. R.; Ceole, L. F.; de Mello J. C. P.; Nakamura, C. V.; Dias Filho, B. P.; Temponi, L. G.; Ueda-Nakamura, T. Antimicrobial activity of plants used as medicinals on an indigenous reserve in Rio das Cobras, Paraná, Brazil. Journal of Ethnopharmacology 2012, 143, 631. [CrossRef] [PubMed]

${ }^{7}$ Nkeh-Chungag, B. N.; Ndebia E. J.; Mbafor, J. T.; Dotwana, L. A.; Oyedeji, O. O.; Iputo, J. E. The effect of Cordia platythyrsa on various experimental models of pain and carrageenan induced inflammation. African Journal of Biotechnology 2014, 13, 343. [CrossRef]

${ }^{8}$ Chauhan, M. G.; Chavan, S. S. Pharmacognosy and biological activity of Cordia rothii Roem. \& Schult. bark. Indian Journal of Traditional Knowledge 2009, 8, 598. [Link]

${ }^{9}$ De Melo, J. I. M.; de Sales, M. F. Boraginaceae A. Juss. na região de Xingó: Alagoas e Sergipe. Hoehnea 2005, 32, 369. [Link]

${ }^{10}$ Moreira, D. L.; Guarim-Neto, G. Usos múltiplos de plantas do Cerrado: um estudo etnobotânico na comunidade sítio Pindura, Rosário Oeste, Mato Grosso, Brasil. Polibotánica 2009, 27, 159. [Link]

11 National Committee For Clinical Laboratory Standarts. Norma M27-A2. 2002. Disponível em: <http://www.anvisa.gov.br $>$. Acesso em: 26 novembro 2012
12 National Committee For Clinical Laboratory Standarts. Norma M7-A6. 2003. Disponível em: $<$ http://www.anvisa.gov.br>. Acesso em: 26 novembro 2014.

${ }^{13}$ Alves, E. G.; Vinholis, A. H. C.; Casemiro, L. A.; Furtado, N. A. J. C.; Andrade e Silva, M. L.; Cunha, W. R.; Martins, C. H. G. Estudo comparativo de técnicas de screening para avaliação da atividade anti-bacteriana de extratos brutos de espécies vegetais e de substâncias puras. Química Nova 2008, 31, 1224. [CrossRef]

${ }^{14}$ Cabral; I. S. R.; Oldoni, T. L. C.; Prado, A.; Bezerra, R. M. N.; de Alencar S. M.; Ikegaki, M.; Rosalen, P. L. Composição fenólica, atividade antibacteriana e antioxidante da própolis vermelha brasileira. Química Nova 2009, 32, 1523. [CrossRef]

${ }^{15}$ Kojima H, Sato, N.; Hatano, A.; Ogura H. Sterol glucosides from Prunella vulgaris. Phytochemistry 1990, 29, 2351. [CrossRef]

16 El-Askary, H. I. Terpenoids from Cleome droserifolia (Forssk.) Del. Molecules 2005, 10, 971. [CrossRef]

17 Saxena, V. K; Albert, S. 6-Sitosterol-3-O-6D-xylopyranoside from the flowers of Tridax procumbens Linn. Journal of Chemical Sciences 2005, 117, 263. [CrossRef]

${ }^{18}$ Vila-Nova, N. S.; Morais, S. M.; Falcão, M. J. C.; Bevilaqua, C. M. L.; Rondon, F. C. M.; Wilson M. E.; Vieira, I. G. P.; Andrade, H. F. Leishmanicidal and cholinesterase inhibiting activities of phenolic compounds of Dimorphandra gardneriana and Platymiscium floribundum, native plants from Caatinga biome. Pesquisa Veterianária Brasileira 2012, 32, 1164. [CrossRef]

${ }^{19}$ De Carvalho, M. G.; Alves, C. C. F.; Werle, A. A.; Braz-Filho R. Metabólitos especiais isolados de Laseguea erecta (Apocynaceae). Revista Brasileira de Farmacognosia 2006, 16, 497. [CrossRef]

${ }^{20}$ Mahato, S. B.; Kundu, A. P. ${ }^{13} \mathrm{C}$ NMR Spectra of pentacyclic triterpenoids - a compilation and some salient features. Phytochemistry 1994, 37, 1517. [CrossRef]

${ }^{21}$ Pyo, M. K.; Lee, Y. Y.; Yun-Choi, H. S. Anti-platelet effect of the constituents isolated from the barks and fruits of Magnolia obovata. Archives of Pharmacal Research 2002, 25, 325. [CrossRef] [PubMed]

22 Jeong, C.-H.; Jeong, H. R.; Choi, G. N.; Kim, D.-O.; Lee, U.; Heo, H. J. Neuroprotective and anti-oxidant effects of caffeic acid isolated from Erigeron annuus leaf. Chinese Medicine 2011, 6, 25. [CrossRef] [PubMed] 
${ }^{23}$ Al-Musayeib, N.; Perveen, S.; Fatima, I.; Nasir, M.; Hussain, A. Antioxidant, anti-glycation and antiinflammatory activities of phenolic constituents from Cordia sinensis. Molecules 2011, 16, 10214. [CrossRef] [PubMed]

${ }^{24}$ Sripathi, S. K.; Gopal, P.; Lalitha, P. Allantoin from the leaves of Pisonia grandis R. Br. International Journal of Pharmacy and Life Sciences 2011, 2, 815. [Link] 